\title{
John NASSICHUK
}

\section{Tyrans et victimes : le pathétique chez Robert Garnier}

Le pathétique dans le théatre de Robert Garnier présente la version remaniée d'une thèse «Nouveau Régime» de Paris X Nanterre. Travaillant à la suite de spécialistes comme Marie-Madeleine Mouflard, dont l'objectif a été de replacer l'œuvre du dramaturge dans un contexte biographique, et de Gillian Jondorf, qui s'est intéressé de façon utile aux thèmes politiques dans les tragédies, Florence Dobby-Poirson cherche à combler une lacune quant à l'analyse proprement littéraire et stylistique de l'œuvre de ce poète majeur. Disons d'emblée que son travail livre de nombreuses analyses intéressantes et utiles, mais que l'attention exclusive sur le texte même de Garnier l'amène souvent à négliger des questions intéressantes de sources et de contexte, qui ne sont guère négligeables pour une étude poétique et rhétorique.

L'ouvrage est divisé en six chapitres, dont le premier constitue un essai général dans lequel l'auteur s'efforce de retracer l'histoire des théories et des usages du pathétique. Implicite dans le titre est le lien présupposé entre l'art rhétorique et la poésie tragique: "De la rhétorique au théâtre. Étude historique du recours à l'émotion». Dobby-Poirson note que, selon Aristote, le théâtre et la rhétorique se rapprochent en ce qu'ils « suscitent tous deux la crainte et la pitié ». La différence réside visiblement dans le fait que la visée de la rhétorique est de caractère utilitaire, alors que la tragédie vise à induire une réaction de plaisir chez l'auditoire. Les traités de rhétorique latins prolongent, et parfois défigurent, la réflexion d'Aristote sur le rôle des passions dans l'éloquence publique. Ainsi, la pensée de Quintilien, comme celle de ses prédécesseurs latins, souligne la portée psychologique des éléments pathétiques de l'éloquence. À la différence de Cicéron et de Sénèque, il croit que, pour émouvoir, l'orateur doit avant tout se montrer ému. Il doit aussi parler de manière à mettre sous les yeux de l'auditoire les événements qu'il relate, afin de mieux l'émouvoir. Sénèque, qui dénonce dans le $D e$ Ira les excès manipulateurs de la passion oratoire, déploie largement ces techniques 
John NASSICHUK, «Tyrans et victimes : le pathétique chez Robert Garnier », @nalyses, hiver 2008

dans ses tragédies, qui laissent transparaittre l'intention didactique de l'auteur stoïcien. Le $\mathrm{XVI}^{\mathrm{e}}$ siècle a hérité de l'ambivalence qui caractérise les positions de l'Antiquité quant à l'usage des passions dans la rhétorique et au théâtre. Utiles pour servir la vérité et inciter à la sagesse, elles deviennent dangereuses lorsqu'on les pousse à l'excès. À la Renaissance, le théâtre devient le domaine privilégié de la représentation des passions à des fins didactiques et morales. Pendant la deuxième moitié du $\mathrm{XVI}^{\mathrm{e}}$ siècle, les dramaturges protestants, comme Bèze, Grévin, Des Masures et Rivaudeau, se démarquent de manière frappante dans la production de tragédies. Ces auteurs se servent de la scène tragique comme lieu de renseignement biblique et d'édification. Dans la théorie, Jean de La Taille identifie le pathétique selon la manière dont il apparait dans la tragédie humaniste: "un principe de plaisir, dont le docere n'est peut-être que l'alibi ». Or, cette revue des théories et pratiques du pathos dramatique, depuis Aristote jusqu'à la fin du $\mathrm{XVI}^{\mathrm{e}}$ siècle, ne dégage pas avec clarté une hypothèse les présupposés effectifs de la pratique de Garnier lui-même. Il dessine un tableau souvent intéressant, mais sans direction, sur le vaste ensemble de prises sur le pathos susceptibles d'informer le théâtre de la fin de la Renaissance.

Le deuxième chapitre examine « le sujet tragique chez Garnier » (p. 97146). Celui-ci privilégie des sujets de tragédie «lamentables» afin de favoriser l'émotion pathétique, ce qui l'amène à mettre en scène «des faits atroces ", souvent des événements illustres déjà mis en scène par les dramaturges de l'Antiquité. Quant aux sources, Euripide est le poète tragique grec le plus souvent cité par Garnier, sans doute en raison de la disponibilité de ses textes en traductions françaises et latines. Dobby-Poirson suit M.-M. Mouflard en affirmant que "Garnier s'intéresse surtout aux pièces grecques où "le lyrisme prédomine", c'est-à-dire celles où l'effusion des sentiments est le plus développé.» (p. 105) Aussi fut-ce sans doute ce penchant pour le lyrisme qui inspira le choix de la matière romanesque dans Bradamante. Comme maint dramaturge au $\mathrm{XVI}^{\mathrm{e}}$ siècle, Garnier insiste sur le caractère exemplaire de l'action qu'il met en scène. Il accorde un statut symbolique particulier à la ville de Troie, dans laquelle se condensent les horreurs de la guerre. Afin d'accroitre l'effet pathétique, le 
John NASSICHUK, «Tyrans et victimes : le pathétique chez Robert Garnier », @nalyses, hiver 2008

dramaturge tend à multiplier les événements funestes, pratique qui l'amène souvent à omettre des passages chez ses modèles qui ne contribuent pas au pathos. "Garnier n'est donc pas esclave de ses sources », explique Dobby-Poirson (p. 132), puisque c'est son souci du pathétique, et non la fidélité de l'imitation textuelle, qui gouverne dans ses pièces le travail inventif. Le jeu des passions et l'enjeu complexe des liens affectifs constituent les principaux lieux de ce travail autant rhétorique que poétique. Si Garnier exhibe « une prédilection pour les sujets de tragédie où les héros et les peuples sont soumis à la pression d'un destin écrasant», ce destin intervient "par l'intermédiaire de passions destructrices » (p. 145). Garnier, conclut l'auteur, « intériorise, partiellement ou totalement, le conflit tragique.»C'est le constat majeur de ce chapitre, qui soutient le caractère psychologique, intérieur, du jeu tragique chez Garnier. Une position critique aussi intéressante aurait bénéficié largement d'analyses comparatives plus nombreuses et plus systématiques, pour définir la spécificité du sujet tragique chez Garnier au sein du corpus dramatique de son époque.

Une "étude de la dramaturgie » constitue la matière du troisième chapitre (p. 147-289). Florence Dobby-Poirson examine le rapport du théâtre de Garnier aux grands principes de l'art dramatique remontant à l'Antiquité grecque: les unités de lieu et de temps, la structure dramatique des pièces, l'occupation de l'espace scénique, les personnages, les types de dénouement tragique. Selon l'auteur, le théâtre de Garnier requiert l'élargissement de «l'unité de lieu » puisque la complexité de l'espace sur la scène remplit chez lui une véritable fonction dramatique. Ainsi, par exemple, la séparation physique permet d'expliquer les tribulations de couples qui ne souffrent pas de réel désaccord (Cléopâtre/Antoine; Roger/Bradamante). De cette manière, l'emploi de l'espace scénique, prévu par Garnier selon les didascalies, approfondit souvent le pathos de l'action. Or, celle-ci, selon Dobby-Poirson, correspond souvent à la célèbre « unité de temps » en raison de la force pathétique des discours et des gestes. Souvent même, l'action se déroule dans un laps temporel qui correspond parfaitement aux exigences du jeu scénique, ce qui renforce l'engagement du spectateur. Chez Garnier, les péripéties servent davantage à susciter la réaction des personnages (et des spectateurs) 
John NASSICHUK, «Tyrans et victimes : le pathétique chez Robert Garnier », @nalyses, hiver 2008

qu'à produire les effets du «choc dramatique », tendance qui l'éloigne d'Aristote quant à la conception du pathétique. "On voit ainsi s'instaurer des rapports entre l'action et l'émotion propres au théâtre de Garnier, et qui se fondent sur un emploi limité mais extrêmement subtil des ressources du "suspens"»(p. 221). Même la structure dramatique des pièces, qui présentent des « systèmes de parallélisme et d'opposition» (p. 223), tendent à renforcer l'effet émotif de chaque situation. Aussi la fréquence des changements de point de vue, qui constitue l'une des principales caractéristiques du théâtre de Garnier, permet-elle au dramaturge d'approfondir la complexité émotive et psychologique des personnages. C'est bien dans cette complexité intérieure que réside, selon Florence Dobby-Poirson, l'originalité dramaturgique de Garnier, dont les personnages tragiques font souvent preuve d'un courage stoïcien. «En intériorisant ainsi l'action de la fatalité, il leur restitue la liberté de comprendre ce qui les a perdus et par là, de prendre en charge leur destinée.» (p. 292). La thèse est séduisante, mais elle est avancée dans l'indépendance absolue de tout rapport sérieusement développé avec les textes antécédents ou contemporains, si bien que les preuves alléguées ne permettent pas de cerner de manière convaincante l'individualité poétique de Garnier.

Le quatrième chapitre comporte une "étude du dialogue» dans le corpus dramatique de Garnier (p. 293-374). Celui-ci, qui avait reçu une formation de juriste, déploie habilement la rhétorique judiciaire dans les dialogues de ses pièces. Lorsqu'il se sert d'antiques modes du dialogue tragique comme la stichomythie, l'ambiguité des répliques tend à " provoquer la crainte et la pitié » (p. 329). Lecteur inventif des sources narratives épiques, Garnier éprouve aussi la «tentation du récit». Dobby-Poirson note de manière intéressante qu'un apport majeur de Garnier à l'art de la tragédie réside dans la modification qu'il impose souvent à cette technique héritée de Sénèque. Alors que celuici présente des séquences narratives qui se déroulent sans interruption sur la scène, Garnier permet aux autres personnages d'intervenir, ce qui augmente naturellement la tension pathétique de ces passages. Enfin, la déploration funèbre constitue l'apogée de l'épanchement sentimental, «grâce à une expression plus distanciée et plus stylisée de la douleur» (p. 359). Garnier cherche à développer le pathétique en 
John NASSICHUK, «Tyrans et victimes : le pathétique chez Robert Garnier », @nalyses, hiver 2008

déployant plusieurs formes de dialogue, qui mettent en œuvre plusieurs modes de représentation.

$\mathrm{Au}$ cinquième chapitre, Florence Dobby-Poirson étudie l'élocution pathétique dans l'œuvre de Garnier, organisant l'évidence textuelle selon trois principales instances discursives: le langage des tyrans, le langage des victimes, l'expression pathétique dans les récits. Du point de vue stylistique, aucun trait constant ne réunit les divers tyrans qui apparaissent dans les pièces de Garnier. C'est plutôt une ressemblance éthique qui les réunit, car chacun à sa manière succombe à une manie de l'excès. Au lieu de relever donc stricto sensu de l'elocutio comme c'est annoncé dans l'introduction du chapitre, cette catégorie conduit plutôt à une réflexion d'ordre thématique, puisque l'auteur conclut à propos des tyrans que "Garnier n'a garde de pousser leur fureur jusqu'à la démence caractérisée » (p. 401) et que «la représentation des passions chez Garnier concorde avec leur description dans les deux Ethiques». Quant aux victimes, elles emploient un lexique semblable à celui de leurs persécuteurs, pour exprimer non la cruauté, mais la répulsion que les tyrans leur inspirent. En effet, l'expression des personnages de Garnier est souvent remarquable pour sa violence. Dobby-Poirson souligne de manière intéressante à cet effet la ressemblance entre cette élocution tragique et la tonalité agressive des pamphlets politiques de l'époque (p. 384-385 et 402). Enfin, le poète tragique déploie à plusieurs occasions l'art du récit afin de souligner le pathétique du souvenir, lorsqu'un personnage affligé remémore son bonheur d'autrefois. Ce chapitre constitue certainement la partie la plus utile et la plus neuve de l'ouvrage, puisqu'il contient de nombreuses analyses originales et précises, qui jettent une vive lumière sur la poétique de Garnier. On peut souligner comme exemple les remarques sur la prosodie pathétique, qui sont d'un intérêt tout particulier (p. 430-435).

Dans le dernier chapitre, intitulé «la diffusion de l'émotion» (p. 535608), l'auteur examine les principaux aspects des pièces qui font du pathétique l'élément central de l'art de la tragédie. Ce chapitre traite successivement des procédés d'amplification, du rapport entre didactisme et pathos, du grand dialogue entre «la vision pathétique de l'homme instable » et la foi religieuse, de l'idée de catharsis dans l'univers 
John NASSICHUK, «Tyrans et victimes : le pathétique chez Robert Garnier », @nalyses, hiver 2008

tragique de Garnier. Dans cette dernière partie, Dobby-Poirson conclut que Garnier partage avec Jean de La Taille une même conception du plaisir tragique. Chez les deux auteurs, "les effets de l'art semblent davantage tendre à exacerber l'émotion qu'à la sublimer » (p. 605). Faire oublier pendant un temps aux spectateurs les bouleversements de leur propre époque, telle semble avoir été en partie la mission des plus grands dramaturges de la période des guerres civiles.

En somme, la force de cet ouvrage réside dans la précision des analyses, qui en maint endroit jettent une lumière éclairante sur le travail de l'un des auteurs majeurs de la seconde moitié du XVI ${ }^{\mathrm{e}}$ siècle. Pour cette raison, il sera utile aux futurs éditeurs des textes de Garnier ainsi qu'à tout chercheur qui s'intéresse à l'écriture du tragédien. Il faut toutefois souligner le fait que ce détail de l'analyse constitue un véritable parti pris critique. La faiblesse relative de la documentation primaire et secondaire limite en effet l'utilité de l'ouvrage aux travaux spécialisés qui traitent de l'esthétique littéraire et du détail poétique. Pour les chercheurs qui souhaitent replacer les œuvres de Garnier dans le contexte critique, politique et historique qui leur appartient, les travaux antécédents, et notamment celui de M.-M. Mouflard, demeurent encore indispensables.

Enfin, une remarque sur la facture du volume, qui aurait bénéficié d'une relecture supplémentaire. On trouve en effet de nombreuses coquilles, dont je me permets de signaler ici quelques-unes : p. $55,1.8$, «la femme bafouée »; p. 67, 1. 5, «d'une certaine douceur »; p. 85, n. 1, 1. 4, « H.W. Lawton »; p. 104, 1. 20, « source de ses tragédies »; p. 196, 1. 6, «Nabuchodonosor se montre»; p. 211, 1. 8, "un moyen de réveiller »; p. 223, 1. 14, "alternativement »; p. 269, 1. 16, «Talthybie »; p. 276, 1. 22, «elles souhaitent la mort»; p. 279, 1. 7, « une femme»; p. $436,1.9$, « un certain nombre de traits ».

Référence : Florence Dobby-Poirson, Le pathétique dans le théâtre de Robert Garnier, Paris, Honoré Champion, coll. «Bibliothèque littéraire de la Renaissance » LXVI, 2006, 672 p. 